\title{
Falsas Memórias e Diferenças Individuais: Um Estudo sobre Fatores de Personalidade e Qualidade da Memória
}

\author{
False Memories and Individual Differences: A Study of Personality Traits \\ and Memory Quality
}

\author{
Carmem Beatriz Neufeld*, ${ }^{a}$, Priscila G. Brust-Renck ${ }^{b}$, Aline Ferreira da Rocha ${ }^{c}$, \\ Mariana Sossella ${ }^{c} \&$ Francisco Heitor da Rosa ${ }^{d, e}$ \\ ${ }^{a}$ Faculdade de Filosofia, Ciências e Letras de Ribeirão Preto da Universidade de São Paulo, Ribeirão Preto, Brasil, \\ ${ }^{b}$ Cornell University, Ithaca, USA, ${ }^{c}$ Faculdade Assis Gurgacz, Cascavel, Brasil, \\ ${ }^{d}$ Universidade Norte do Paraná, Londrina, Brasil \& ${ }^{e}$ Universidade Pitágoras, Londrina, Brasil
}

\begin{abstract}
Resumo
As distorções mnemônicas têm recebido atenção crescente da comunidade científica nacional e internacional. No entanto, a relação entre os mecanismos responsáveis pelas distorções mnemônicas e as diferenças individuais no desempenho da memória ainda permanecem pouco explorados na literatura. O presente estudo visa a investigar a influência de fatores de personalidade na suscetibilidade às falsas memórias. Para isso, 200 estudantes universitários de diversos cursos de graduação, foram submetidos ao procedimento de Palavras Associadas (DRM) para investigar o desempenho da memória, e ao Inventário Fatorial de Personalidade (IFP). Os níveis extremos obtidos no IFP (baixa vs. alta pontuação na escala) foram utilizados para investigar as diferenças individuais. Os resultados mostraram que memórias verdadeiras e falsas foram afetadas apenas pelos fatores de personalidade afiliação e autonomia.

Palavras-chave: Falsas memórias, diferenças individuais, reconhecimento de palavras.
\end{abstract}

\begin{abstract}
Research on mnemonic distortions has increased among researchers worldwide. However, the relation between the mechanisms responsible for memory distortions and individual differences that influence memory performance is still to be investigated. The present study aims to investigate the influence of personality traits in the susceptibility to false memories. Participants were 200 college students from different majors. The Deese-Roediger-McDermott (DRM) Procedure was used to investigate memory performance and the Personality Traits Inventory was used to identify distinct personality traits. Results indicate that true and false memories were affected only by affiliation and autonomy personality traits.

Keywords: False memories, individual differences, word recognition.
\end{abstract}

$\mathrm{O}$ fato do ser humano ser capaz de lembrar, de forma espontânea ou induzida, de eventos que nunca aconteceram na sua vida, tem nos instigado a questionarmos os limites entre o falso e o verdadeiro. Essas lembranças de situações que nunca aconteceram, ou que aconteceram de forma diferente da qual foram recuperadas, consistem nas falsas memórias (Neufeld, Brust, \& Stein, 2010; Reyna \& Brainerd, 1995).

Todo ser humano tem a capacidade de memorizar e, portanto, é esperado que erros aconteçam nesse processo
(Stein \& Neufeld, 2001). Por exemplo, as recordações sobre uma palestra, um filme, ou até mesmo de algum evento estressante como um acidente de carro, podem não retratar com fidedignidade a realidade vivenciada. Em alguns casos, essas lembranças errôneas podem até mesmo resultar em conseqüências sérias, afetando diretamente a vida dos indivíduos, como em situações de conflito de relacionamentos amorosos, quando uma pessoa afirma veementemente ter dito algo, quando na verdade, apenas pensou em fazê-lo.

Mas será que alguns indivíduos são mais propensos que outros a lembrarem equivocadamente uma situação que não aconteceu? Gallo (2010), em uma revisão sobre o impacto das pesquisas sobre falsas memórias nos últimos 15 anos, aponta que ainda não foi possível esclarecer quais características individuais são responsáveis pela formação das falsas memórias. A pergunta de que tipos de indivíduos são particularmente suscetíveis ou resistentes 
às falsas memórias ainda é de difícil resposta por ter sido pouco abordada (Barbosa, Ávila, Feix, \& Grassi-Oliveira, 2010; Barrett, Tugade, \& Engle, 2004). Alguns estudos recentes vêm fazendo novas descobertas sobre o papel das diferenças individuais na suscetibilidade a distorções de memória, relacionando alguns traços de personalidade a uma maior tendência a erros de memória (Drivdahl \& Zaragoza, 2001; Peiffer \& Trull, 2000).

A personalidade, segundo a Terapia Cognitivo-Comportamental, refere-se a padrões característicos de pensamento, emoção e comportamento. Esses padrões englobam formas de interpretar o mundo, julgar e tomar decisões e, conseqüentemente, de nos relacionarmos com o mundo e conosco mesmos (Knapp \& Beck, 2008). De acordo com os autores, as pessoas possuem um sistema de crenças, o qual influencia na formação de sua personalidade, assim como os aspectos biológicos como o temperamento. Em outras palavras, cada indivíduo tem um modelo próprio de habilidades, crenças, comportamentos e traços de personalidade que o torna único.

Algumas dessas tendências pessoais parecem estar relacionadas com a suscetibilidade à produção de falsas memórias, como humor deprimido e outros estados emocionais. Com grupos clínicos, Stein e Pergher (2001), ao comparar participantes deprimidos com não deprimidos, encontraram que, comparativamente, estes eram menos suscetíveis a falsas memórias do que aqueles. Estudos com vítimas de traumas têm demonstrado que existe uma diferença importante na recuperação de informação verdadeira e falsa. Um estudo brasileiro, por exemplo, apontou que participantes com e sem história de negligência emocional demonstraram um padrão de resposta semelhante para memória verdadeira, mas as pessoas que sofreram negligência emocional obtiveram menor índice de falsas memórias para listas de palavras (e.g., pânico, escuro) semanticamente relacionadas com conteúdo emocional negativo (e.g., medo) e neutro (e.g., pátria, símbolo estão associadas à palavra bandeira; Grassi-Oliveira, Gomes, \& Stein, 2011). Neste mesmo estudo, observou-se que as palavras negativas foram mais recuperadas do que as palavras neutras pelos dois grupos. Os autores destacam que a diferença entre os grupos, no que se refere às falsas memórias, estaria relacionada à sensibilidade de detecção da essência do material.

O Procedimento de Palavras Associadas (Roediger \& McDermott, 1995; Stein, Feix, \& Rohenkohl, 2006) envolve a apresentação de listas de palavras semanticamente associadas a um mesmo tema (e.g., pânico, escuro) e subseqüente teste de memória. A lembrança de palavras que estavam na lista constitui uma memória verdadeira, enquanto a lembrança equivocada da palavra à qual a lista estava associada, mas que não foi apresentada originalmente (e.g., medo) constitui uma falsa memória.

$\mathrm{O}$ aumento da recuperação de memórias negativas em comparação a neutras também pode ser observado em estudos com indivíduos com Transtorno de Estresse Pós-Traumático (Zoellner, Foa, Brigidi, \& Przeworski,
2000). Os autores avaliaram a memória de pessoas com e sem o transtorno, mas que sofreram trauma. Os resultados indicaram que sofrer trauma é preditor de suscetibilidade a falsas memórias.

Já Salthouse e Siedlecki (2007) compararam a qualidade da memória de participantes saudáveis, usando listas de palavras associadas, a seus escores em uma escala de depressão, um inventário de ansiedade-traço, uma escala para avaliação do afeto negativo e positivo, e o Inventário de Cinco Grandes Fatores de Personalidade. Não foram encontradas relações entre os tipos de memória e os cinco fatores de personalidade. Convém notar, no entanto, que no modelo utilizado pelos autores não foram observadas as subescalas de cada fator, como ocorre no estudo brasileiro (Ávila \& Stein, 2006) e nos estudos internacionais (Gudjonsson, 1983; Peiffer \& Trull, 2000; Salthouse \& Siedlecki, 2007).

Utilizando o mesmo inventário de personalidade que Salthouse e Siedlecki (2007) para medir uma característica específica da escala - o neuroticismo -, Gudjonsson (1983) observou, em um contexto clínico, que indivíduos do sexo masculino com alto índice de neuroticismo produziam um número maior de falsas memórias. Indivíduos que apresentam alto resultado em neuroticismo geralmente caracterizam-se por serem preocupados, nervosos, emotivos, inseguros, inadequados e hipocondríacos. Anteriormente, Peiffer e Trull (2000) haviam optado por realizar um estudo com sujeitos do sexo feminino, também em contexto clínico e com relato de abuso sexual, encontrando relação entre níveis de condescendência e a produção de falsas memórias. Condescendência é a tendência do sujeito a concordar com afirmações, independentemente de seus conteúdos serem divergentes. Segundo os autores, a opção por utilizar sujeitos do sexo feminino se deu porque são o maior contingente clínico e os que mais relatam abuso sexual.

Ávila e Stein (2006) apresentaram resultados semelhantes ao testarem a memória de adultos jovens (com níveis alto, baixo e padrão de neuroticismo) através de listas de palavras semanticamente associadas. Os dados evidenciaram que participantes com altos índices de neuroticismo foram mais suscetíveis a falsas memórias. As autoras foram além e observaram a memória para palavras com conteúdo emocional positivo, negativo e neutro. Os resultados indicaram que participantes com altos níveis de neuroticismo apresentavam mais falsas memórias para palavras com conteúdo negativo em comparação com participantes que apresentavam nível médio desse fator de personalidade.

A importância desses estudos está relacionada à interpretação de resultados de falsas memórias, que devem ser considerados diferentemente conforme as dificuldades cognitivas dos indivíduos. Considerando que características individuais realmente exerçam influência sobre a suscetibilidade à distorção mnemônica, generalizar um resultado independentemente dos traços de personalidade dos participantes parece ser um erro de avaliação. 
Neufeld, C. B., Brust-Renck, P. G., Rocha, A. F., Sossella, M. \& Rosa, F. H. (2013). Falsas Memórias e Diferenças Individuais: Um Estudo sobre Fatores de Personalidade e Qualidade da Memória.

A literatura atual sobre diferenças individuais aponta resultados inespecíficos com relação ao desempenho da memória e suas distorções especialmente por uma falta de padronização dos instrumentos utilizados em pesquisa. A diversidade de medidas de personalidade parece ser um dos impeditivos fundamentais para maiores avanços dos estudos nesta área uma vez que dificulta a comparação dos resultados (Barbosa et al., 2010). No Brasil, Pervin e John (2004) enfatizam que essa dificuldade é ainda maior em função da necessidade de adequação dos instrumentos.

$\mathrm{Na}$ tentativa de investigar a influência das diferenças individuais no desempenho da memória, especialmente no que tange às falsas memórias, o presente estudo optou pela utilização de um instrumento de avaliação da personalidade desenvolvido no Brasil: o Inventário Fatorial de Personalidade - IFP (Pasquali, Azevedo, \& Ghesti, 1997). Além de ter sido inteiramente desenvolvimento no Brasil, o IFP é um questionário extenso que avalia 15 subfatores formadores da personalidade. Sua validação e padronização permitem que a avaliação das respostas seja feita de forma específica conforme o sexo de cada participante, correção coerente com uma teoria que considera cada aspecto das diferenças individuais de cada pessoa. Cabe aqui ressaltar que o IFP não é um instrumento utilizado para avaliação de psicopatologias, embora seus índices extremos (altos ou baixos) sejam bons indicadores de possíveis sintomas ou psicopatologias (ou do desenvolvimento/vulnerabilidade desses sintomas). Portanto, sua sensibilidade na avaliação de traços de personalidade permite uma compreensão dos índices extremos de cada subfator como características que sugerem uma disfuncionalidade dos indivíduos naquele fator em específico.

Além do IFP (Pasquali et al., 1997) ser um instrumento baseado em outro modelo teórico (Modelo de Necessidades de Murray) do que a escala de neuroticismo (Modelo Cinco Grandes Fatores), trata-se de um instrumento brasileiro e que investiga de forma bastante abrangente a personalidade. Tal abrangência do instrumento de medida torna-o interessante para testar sua interação com o padrão de distorções mnemônicas. Cabe ressaltar ainda que não há dados na literatura sobre os traços medidos pelo IFP e as falsas memórias, tornando esta uma lacuna a ser investigada. Além disso, a literatura tem indicado associação entre altos índices de neuroticismo e distorções mnemônicas, no entanto, neste modelo não são encontrados dados sobre os índices extremos baixos de uma determinada característica de personalidade e sua influência sobre a suscetibilidade aos erros de memória. Neste sentido, o IFP poderá auxiliar na investigação tanto dos fatores extremos altos e baixos de personalidade.

A hipótese principal a ser testada neste estudo pressupõe que pessoas com indicadores extremos nos traços de personalidade (que teriam alguns fatores muito acentuados - baixo ou alto) seriam mais suscetíveis às falsas memórias. Essa hipótese vai ao encontro dos estudos de Ávila e Stein (2006) e de Gudjonsson (1983) que consideram que indiví- duos com níveis disfuncionais dos traços de personalidade, como altos níveis de neuroticismo, são mais suscetíveis à falsificação da memória. Além disso, por meio do presente trabalho espera-se observar diferenças na recuperação de informações com conteúdo emocional negativo, positivo e neutro quando determinados fatores de personalidade predominam ou se encontram abaixo da média.

Considerando a literatura e tomando como base os perfis cognitivos descritos em Terapia Cognitivo-Comportamental (Beck, 1971; Beck, Freeman, \& Davis, 2005), poder-se-ia hipotetizar que os indivíduos excessivamente voltados para os outros (como por exemplo, baixa autonomia, alta afiliação, e alto afago) seriam mais suscetíveis a distorções de palavras de conteúdo emocional positivo, uma vez que os mesmos tenderiam a lembrar dos aspectos reforçadores das suas relações sociais, devido a sua necessidade constante de apoio nas relações com os outros. Neste mesmo sentido, indivíduos altamente independentes e rígidos e que interpretem as relações sociais mais como fonte de privação do que de prazer (por exemplo, com alta dominância, alta autonomia e baixa mudança) tenderiam a maior distorção de palavras de conteúdo emocional negativo, uma vez que os mesmos teriam um perfil similar ao descrito em pessoas com altos índices de neuroticismo e seu foco estaria voltado para os aspectos negativos das relações com os outros.

\section{Método}

\section{Participantes}

Participaram do estudo 200 universitários de diferentes cursos (Administração, Ciências Contábeis, Enfermagem, Engenharia Civil, Farmácia, Gestão de Vendas, Letras, Nutrição, Pedagogia, Psicologia, entre outros) de Instituições Privadas de Ensino Superior do interior do Paraná. A faixa etária dos participantes foi de 18 a 48 anos $(M=23,3, D P$ $=5,86$ ), sendo 114 do sexo feminino e 86 do masculino, os quais foram selecionados por conveniência.

\section{Instrumentos}

O desempenho da memória foi investigado por meio da utilização do Procedimento de Palavras Associadas adaptado para a população brasileira por Stein et al. (2006) e utilizado integralmente conforme descrito pelos mesmos autores. O procedimento envolveu a apresentação de listas de palavras semanticamente associadas a um mesmo tema. Foram utilizadas oito listas de 10 palavras (e.g., dedos, sapatos, unha, chulé, meia, calçado, tênis, sustentação, chinelo, calo), todas as quais estão associadas a uma palavra não apresentada (nesse caso, pé). As listas variavam em termos de conteúdo emocional, sendo duas negativas (raiva e mágoa), duas positivas (alegria e satisfação), e quatro neutras (alto, áspero, pé, xícara). As normas de valência da lista foram extraídas de Stein e Gomes (2009; Tabela 3), com escores baixos (de 1 a 3,99$)$ para mágoa $(M=1,70$; $D P=1,70)$ e raiva $(M=1,60, D P=1,54)$, escores altos (de 7 a 9) para alegria $(M=8,62 ; D P=1,30)$ e satisfação 
$(M=8,52 ; D P=1,35)$, e escores médios (de 4 a 6,99$)$ para alto $(M=5,13 ; D P=1,87)$, áspero $(M=4,12 ; D P=$ $2,22)$, pé $(M=4,72 ; D P=2,19)$, e xícara $(M=6,21 ; D P=$ $2,39)$. A classificação dos itens em valência negativa, positiva, e neutra foi extraída da compreensão de Kensinger e Corkin (2004) bem como de Santos, Silveira, Gomes e Stein (2009) para o entendimento das reações emocionais segundo o material utilizado em Stein e Gomes (2009).

Cada palavra foi apresentada auditivamente em intervalos de 2 segundos. O teste de memória de reconhecimento para as listas apresentadas foi composto por 48 itens, dos quais 24 eram itens-alvo (medida de memórias verdadeiras), oito eram distratores críticos (medida de falsas memórias), e 16 eram distratores não-relacionados (medida de respostas não mnemônicas ou chute). As palavras do teste de reconhecimento foram aleatorizadas em uma mesma ordem para todos os participantes. Os participantes foram instruídos a assinalar sim ou não ao lado de cada palavra do teste conforme suas lembranças a respeito das palavras que estavam na lista inicial.

As diferenças individuais foram avaliadas por meio do Inventário Fatorial de Personalidade - IFP (Pasquali et al., 1997). Este Inventário é composto por 155 frases, sendo 135 acerca de 15 fatores de personalidade e 20 referentes às escalas de desejabilidade social e validade, com o intuito de verificar a confiabilidade das respostas. Os participantes foram instruídos a ler cada uma das frases e assinalar o quanto aquela informação representa uma característica de sua personalidade em uma escala Likert de 1 (nada característico) a 7 (totalmente característico).

Os 15 fatores avaliados pelo IFP referem-se à assistência (desejo de auxiliar pessoas indefesas ou em situação de necessidade), intracepção (se deixar conduzir por sentimentos e inclinações difusas, sendo dominado pela procura da felicidade, pela fantasia e imaginação, além de julgar as outras pessoas por suas reais ou supostas intenções, não tanto pelos atos em si), afago (necessidade de receber afeto de pessoas queridas), deferência (admirar e apoiar um superior), afiliação (ter prazer em contribuir com um aliado), dominância (controlar o ambiente humano), denegação (aceitar críticas sem reagir), desempenho (obter reconhecimento pelas tarefas executadas), exibição (desejo de fascinar, impressionar ou entreter as pessoas), agressão (superar a oposição pela força ou depreciação do outro), ordem (necessidade de precisão, organização e limpeza), persistência (capacidade de finalizar empreendimentos), mudança (agrado pelo diferente, novo ou exótico), autonomia (libertar-se de restrições, agir por conta própria) e heterossexualidade (desejo de relacionar-se afetiva ou sexualmente com o sexo oposto).

\section{Delineamento}

A fim de verificar a influência das características de personalidade na suscetibilidade às falsas memórias, $o$ presente estudo utilizou um delineamento fatorial $3 \times 3 \times$ 2, com medidas repetidas para todas as variáveis. As duas primeiras variáveis se referem aos itens do teste de memória, que podem variar de acordo com a emocionalidade (positiva, neutra, e negativa) ou tipo de item (alvo, distrator crítico, e distrator não-relacionado). A última variável diz respeito aos fatores de personalidade, que foram considerados em seus dois níveis extremos de característica (alto ou baixo). A variável dependente foi o desempenho da memória no teste de reconhecimento.

\section{Procedimento}

Após a aprovação do estudo pelo Comitê de Ética em Pesquisa com Seres Humanos, a coleta de dados foi realizada em grupos de aproximadamente 20 alunos, em sala silenciosa. Foram explicados todos os procedimentos aos participantes, bem como assegurados os critérios éticos da pesquisa. Todos os participantes que assinaram o Termo de Consentimento Informado, Livre e Esclarecido participaram da pesquisa. Inicialmente foi apresentado aos participantes o Procedimento de Palavras Associadas. Em seguida, os participantes responderam o Inventário Fatorial de Personalidade - IFP. Por fim, quando o IFP foi completado por todos os participantes de cada grupo (o tempo gasto pelos sujeitos de pesquisa para responder ao IFP manteve-se entre $40 \mathrm{~min}$ e $50 \mathrm{~min}$ ), foi realizado o teste de memória de reconhecimento.

\section{Resultados e Discussão}

Os dados foram analisados com o auxílio do programa Statistical Package for Social Sciences (SPSS) versão 15.0. Todos os tratamentos estatísticos utilizaram um $\alpha=0,05$ para o teste de hipóteses. Foram realizadas análises post hoc e comparações pareadas com correção de Bonferroni.

\section{Indicadores de Memória}

A avaliação dos dados do teste de reconhecimento foi realizada de forma que a recuperação de itens-alvos representava a medida de memória verdadeira (MV), a de distratores críticos, a medida de falsas memórias (FM), e a de distratores não-relacionados, a respostas não mnemônicas (RNM).

Considerando-se os resultados de memória geral, as respostas para o teste de memória foram analisadas através de uma Análise de Variância (ANOVA) para os três tipos de item do teste de memória $[F(2,398)=1138,70, p$ $<0,001]$. Os resultados evidenciaram que os participantes obtiveram médias superiores de FM $(M=0,72, D P=0,19)$ do que de $\operatorname{MV}(M=0,68, D P=0,15 ; p<0,001)$, o que corrobora os resultados de estudos de Ávila e Stein (2006) utilizando o mesmo procedimento. Segundo Roediger e McDermott (1995), índices superiores de lembranças falsas do que verdadeiras podem ser esperadas para o Procedimento de Palavras Associadas quando se utiliza um teste de reconhecimento. Já as RNM $(M=0,14, D P$ $=0,15$ ), que são a medida de esquecimento ou chute uma vez que as mesmas não têm base na memória para as 
Neufeld, C. B., Brust-Renck, P. G., Rocha, A. F., Sossella, M. \& Rosa, F. H. (2013). Falsas Memórias e Diferenças Individuais: Um Estudo sobre Fatores de Personalidade e Qualidade da Memória.

palavras apresentadas no estudo, apresentaram médias inferiores tanto a FM como a MV ( $p$ s $<0,001)$, o que indica uma boa qualidade da memória dos participantes, semelhante ao resultado encontrado por Stein et al. (2006). Como a função das RNM era verificar o nível de chute das respostas do teste, seu baixo índice de aceitação permite que sejam excluídas das análises posteriores, uma vez que sua presença é utilizada como uma forma de controle, no intuito de verificar se os sujeitos responderam o teste de memória com base no material alvo. Além disso, no material de Stein et al. (2006) utilizado no presente estudo, bem como nas normas descritas por Santos et al. (2009) e Stein e Gomes (2009), as RNM não são divididas quanto a sua emocionalidade, impedindo, portanto análises das RNM em termos de sua carga emocional. Neste sentido, os resultados nas seções seguintes não incluirão análises de RNM, apenas de MV e FM.
Esses resultados foram explorados em uma ANOVA para sexo (feminino vs. masculino) $\mathrm{x}$ tipo de item do teste de memória (itens-alvo vs. distratores críticos) x emocionalidade da lista (positiva vs. negativa vs. neutra), com medidas repetidas nas duas últimas variáveis. Esta análise evidenciou efeito principal apenas para a variável tipo de item $[F(1,199)=18,73, p<0,001]$, com dados semelhantes ao resultado anterior, portanto não será documentado. Cabe ressaltar que não foram observadas diferenças na memória entre homens e mulheres, assim os grupos foram considerados equivalentes, e essa variável foi excluída das próximas análises. Os resultados, portanto mostram-se contrários aos estudos de Gudjonsson (1983) e de Peiffer e Trull (2000).

O efeito principal da ANOVA foi qualificado por uma interação entre tipo de item do teste e emocionalidade da lista $[F(2,398)=30,10, p<0,001]$ destacado na Tabela 1 .

Tabela 1

Média de Reconhecimento por Tipo de Item e Emocionalidade da Lista

Emocionalidade da lista

\begin{tabular}{lccc}
\cline { 2 - 4 } \multicolumn{1}{c}{ Tipo de item } & Positiva & Negativa & Neutra \\
\hline Alvos & $0,62(0,26)$ & $0,74(0,23)^{* *}$ & $0,69(0,18)^{* *}$ \\
Distratores críticos & $0,79(0,33)^{*}$ & $0,68(0,27)$ & $0,73(0,27)$ \\
\hline
\end{tabular}

Nota. Desvio padrão entre parênteses.

$* p<0,05$ entre emocionalidade da lista; $* * p<0,001$ entre emocionalidade da lista.

Os dados sugerem uma relação inversamente proporcional entre a recuperação de informações positivas e negativas, de forma que a recuperação das palavras com conteúdo negativo foi superior à de conteúdo positivo em termos de MV $(p<0,001)$ e inferior em termos de $\mathrm{FM}(p<0,001)$. Com relação às informações neutras, os resultados apenas ressaltam uma recuperação verdadeira superior em comparação às palavras positivas e inferior em relação às negativas $(p s<0,001)$. Os resultados para memórias verdadeiras corroboram os achados da literatura: informações negativas são mais bem lembradas que informações positivas e neutras (Ávila \& Stein, 2006; Brainerd, Stein, Silveira, Rohenkohl, \& Reyna, 2008). Os resultados para falsas memórias, no entanto, diferem dos achados anteriores, que mostraram mais distorções para informações negativas.

\section{Indicadores de Personalidade}

Os fatores de personalidade do IFP foram categorizados conforme o grau da característica que uma informação representa, e apenas avaliados nos seus níveis extremo baixo (de percentil 5 a 25) e extremo alto (de 75 a 100). Essa divisão foi realizada no intuito de observar apenas aqueles participantes com níveis muito marcantes de algum traço de personalidade, uma vez que, segundo as normas do teste os níveis intermediários dos fatores representam um funcionamento típico da personalidade. Todos os participantes apresentaram pelo menos um dos fatores em nível extremo.

Para a investigação da influência dos fatores de personalidade foi realizada uma ANOVA para cada característica de personalidade em seus dois níveis extremos (alto vs. baixo) com medidas repetidas para o tipo de item do teste de memória (itens-alvo vs. distratores críticos) $\mathrm{x}$ emocionalidade da lista (positiva vs. negativa vs. neutra) controlando para desejabilidade social (DS) ${ }^{1}$. Como DS não se refere a um fator de personalidade e sim a uma escala de controle do instrumento, participantes com altos índices desse fator (índice percentílico maior ou igual a 75) foram excluídos das análises. A DS está diretamente relacionada à tentativa dos participantes de agradar o pesquisador ao emitir suas respostas, sendo esta uma das possíveis fontes de erros de medida em testes psicológicos apontada por Pasquali (2000). Portanto, as análises subseqüentes foram realizadas para os 134 participantes sem características extremas de DS.
1 Quando o fator desejabilidade social é levado em consideração na ANOVA, somente o fator "afiliação" interage com variáveis de memória. 
Somente serão apresentados os resultados dos fatores de personalidade que indicarem uma interação entre os fatores da ANOVA (ver Tabela 2). As análises indicaram interações marginalmente significativas entre o desempe- nho da memória para informações emocionais e os fatores de personalidade afiliação $[F(2,84)=2,86, p=0,06] \mathrm{e}$ autonomia $[F(2,57)=2,72, p=0,08]$.

Tabela 2

Média de Reconhecimento por Tipo de Item e Emocionalidade da Lista para os Fatores de Personalidade Afiliação e Autonomia em seus Níveis Extremos (alto e baixo) Controlado para Desejabilidade Social

Fatores de personalidade

Tipo de item

Emocionalidade

da lista

Afiliação Autonomia

\begin{tabular}{lccccc}
\cline { 3 - 5 } & & Baixo & Alto & Baixo & Alto \\
\hline \multirow{2}{*}{ Alvos } & Positiva & $0,47(0,23)$ & $0,65(0,27)$ & $0,58(0,26)$ & $0,59(0,25)$ \\
& Negativa & $0,80(0,15)^{*}$ & $0,74(0,24)^{*}$ & $0,76(0,22)^{*}$ & $0,67(0,28)$ \\
& Neutra & $0,70(0,18)$ & $0,66(0,18)$ & $0,68(0,17)$ & $0,67(0,17)$ \\
& Positiva & $0,75(0,26)$ & $0,77(0,35)$ & $0,84(0,29)^{*}$ & $0,74(0,30)$ \\
& Negativa & $0,60(0,26)$ & $0,69(0,26)$ & $0,60(0,28)$ & $0,68(0,25)$ \\
& Neutra & $0,77(0,16)$ & $0,74(0,26)$ & $0,70(0,29)$ & $0,74(0,31)$ \\
\hline
\end{tabular}

Nota. Desvio padrão entre parênteses.

$* p<0,05$ entre emocionalidade da lista.

Os resultados das interações para o fator afiliação demonstraram que indivíduos com níveis tanto baixos como altos de afiliação lembraram mais informações verdadeiras com conteúdo emocional negativo do que positivo ( $p$ s $<0,05)$, e que indivíduos com baixos níveis do fator recuperaram mais informações verdadeiras com conteúdo neutro do que com conteúdo positivo $(p=0,06)$. Ainda, informações verdadeiras com conteúdo neutro foram menos lembradas do que com conteúdo negativo $(p s<0,05)$ por participantes com níveis altos de afiliação. Não foram observadas diferenças para as falsas memórias.

Uma importante diferenciação entre os níveis alto e baixo do fator afiliação (ver Tabela 2) pode ser observada na recuperação de informações verdadeiras com conteúdo emocional positivo, uma vez que foram mais lembradas pelos participantes com níveis altos deste fator $(p<0,05)$. Tal resultado indica que participantes com alta afiliação lembram mais informações verdadeiras que tenham conteúdo emocional positivo associado, o que parece condizente com um padrão de comportamento de pessoas que têm necessidade de estreitar laços já que as informações emocionalmente carregadas normalmente estão associadas ao estabelecimento e manutenção de relações interpessoais. Esse resultado parece reforçar também a disfuncionalidade do traço de personalidade afiliação baixo, visto que a dificuldade de lembrar informações positivas pode influenciar na dificuldade de manter laços afetivos.
Em geral, tais resultados sugerem que tanto ter dificuldade em fazer, manter e estreitar laços afetivos com grupos de relacionamento (baixa afiliação) quanto ter necessidade exagerada de sentir-se como pertencente a um grupo e ter relação de laços estreitada com várias pessoas chegando por vezes à indiferenciação em relação a este grupo (alta afiliação), exerce efeitos similares em termos de lembranças verdadeiras de palavras emocionalmente negativas. No caso dos baixos índices deste fator, tal direcionamento da memória pode retroalimentar a dificuldade do indivíduo de afiliar-se, uma vez que ele tende a lembrar mais de conteúdos negativos e neutros de suas experiências e esquecer conteúdos positivos. Já no caso dos indivíduos com altos índices de afiliação, este padrão de lembrança de mais informações negativas nivelaria todos os componentes de um grupo (mesmo que negativamente) e permitiria ao sujeito evitar a necessidade de fazer escolhas dentro do grupo, simpatizando com uns e antipatizando com outros, justificando sua necessidade de manter-se afiliado independente das reações dos componentes do grupo.

No que tange ao traço de personalidade autonomia (ver Tabela 2), as diferenças marginalmente significativas ocorreram nos índices de memória dos participantes com níveis extremos baixos de autonomia. Participantes com baixa autonomia têm dificuldade de tomar decisões e de gerenciar suas próprias vidas, de agir por conta própria. Comumente necessitam de constantes incentivos e reasseguramentos, $\mathrm{e}$ 
Neufeld, C. B., Brust-Renck, P. G., Rocha, A. F., Sossella, M. \& Rosa, F. H. (2013). Falsas Memórias e Diferenças Individuais: Um Estudo sobre Fatores de Personalidade e Qualidade da Memória.

não poucas vezes apresentam características de inatividade. A exemplo dos resultados obtidos pelos participantes com índices extremos baixos de afiliação, os participantes com baixa autonomia recordaram mais informações verdadeiras negativas do que positivas $(p<0,05)$. Em relação à produção de falsas memórias, as informações positivas foram mais distorcidas do que negativas $(p<0,05)$. Esse resultado não corrobora os dados de memória do presente estudo e de Ávila e Stein (2006), que ressaltam que, em geral, são lembradas mais informações verdadeiras e falsas com conteúdo negativo do que positivo por indivíduos com alto nível de neuroticismo.

A discordância dos resultados do presente estudo com a literatura pode repousar na diferença entre as matrizes teóricas utilizadas para avaliar as características de personalidade ou, até mesmo no nível de disfuncionalidade dos indivíduos. Ávila e Stein (2006) observaram essa tendência de respostas numa faixa da amostra que seria classificada, tipicamente, como disfuncional de acordo com o modelo dos Cinco Grandes Fatores. Em contrapartida, os grupos extremos identificados pelo IFP não são considerados, necessariamente, disfuncionais, apesar de indicar uma maior vulnerabilidade ao desenvolvimento de sintomas. Além disso, indivíduos com altos índices de neuroticismo não são comumente pouco autônomos, pelo contrário, em geral são descritos como indivíduos com dificuldade de considerar opiniões alheias e de serem inflexíveis em relação a suas próprias opiniões, não estando disponíveis para rever posturas tipicamente adotadas.

\section{Considerações Finais}

O principal objetivo do presente estudo foi investigar a relação entre fatores de personalidade e a suscetibilidade às falsas memórias. Observou-se que algumas características individuais marcantes da vida dos indivíduos interagiram com a falsificação de mais informações do que outras. O nível de característica que um fator representava para os indivíduos também se mostrou responsável pelos reconhecimentos verdadeiros e falsos conforme o conteúdo emocional positivo e negativo de uma informação. Portanto, pode-se pensar que pessoas com características marcantes de personalidade (que teriam algumas características em nível extremo alto ou extremo baixo) possuiriam predisposição a ter um desempenho diferenciado, positiva ou negativamente, de memória, especialmente quando a informação a ser recuperada possui conteúdo emocional.

Os resultados desse estudo levantam importantes implicações para a Psicologia Jurídica e a Clínica, uma vez que a personalidade de testemunhas, vítimas e pacientes pode sofrer influência na fidedignidade da recuperação de uma informação. Ou seja, algumas características de personalidade podem levar o indivíduo a relatar a situação real de forma diferente da realidade vivenciada, por lembrar-se de forma distorcida o evento.

$\mathrm{O}$ presente estudo sugere que índices extremos altos e baixos de afiliação e extremo baixo de autonomia parecem interagir com a fidedignidade da memória para palavras emocionais. Tais dados necessitam de aprofundamento em estudos posteriores, uma vez que a combinação de alto índice de afiliação e baixo índice de autonomia, por exemplo, é característica marcante de transtornos mentais como o transtorno de personalidade dependente. Dados como este poderiam levar a futuros estudos com população clínica no intuito de investigar de forma mais sistemática uma relação entre determinados transtornos mentais e a suscetibilidade à distorção.

No que se refere à desejabilidade social (DS) também parece ser necessário uma maior investigação. Apesar de DS não ser tratada neste modelo de personalidade como uma característica em particular, em função dos dados obtidos torna-se essencial verificar mais amplamente o efeito da desejabilidade social nos processos de memória. Fica claro que, mesmo em pequenas amostras, é possível visualizar o impacto desta variável em diferentes circunstâncias, o que torna a inclusão da variável como necessária em estudos desta natureza. Os resultados deste grupo de análise sugerem que DS talvez seja um fator explicativo da performance da memória, uma vez que afiliação e autonomia podem ter relação com maior ou menor necessidade de "agradar" os outros.

Nesse mesmo sentido, considerando a implicação desta variável sobre amostras clínicas, alguns transtornos parecem ter maior preocupação em se posicionar de forma desejável frente ao social. Portadores de transtorno de ansiedade social, por exemplo, preocupam-se de forma extremada com a impressão que suas ações causam nas outras pessoas. Tais preocupações podem influenciar as suas memórias sobre eventos sociais posteriores, impactando sobre o aumento ou a diminuição de seus próprios sintomas de ansiedade em situações sociais futuras. Sugere-se que estudos adicionais sejam feitos no sentido de explicar o papel da DS no processo de fidedignidade da memória.

Além disso, algumas limitações podem ser apontadas no presente estudo, como o tamanho da amostra. Por ser um estudo que envolve a investigação de características de personalidade, obteve-se uma amostra por conveniência, não havendo quantidade semelhante de indivíduos distribuídos em cada fator. O número de fatores de personalidade envolvidos também indica a necessidade de uma amostra maior para fins de análises estatísticas. Outro aspecto que pode ser considerado como limitação no presente estudo refere-se ao fato de que não foi controlado o tempo de intervalo entre a apresentação do material alvo e o teste de memória. Apesar de o intervalo ter se mantido entre 40 min e 50 min, esta variação pode ter gerado algum ruído nos dados, e deveria ser controlado em estudo futuros.

Os resultados encontrados sugerem que novos estudos são necessários e que devem indicar de maneira mais clara e precisa a relação entre características de personalidade e a suscetibilidade à distorção mnemônica em situações cotidianas. $\mathrm{O}$ fato de o estudo ter utilizado listas de palavras pode também gerar influência sobre a ativação ou não de um estilo cognitivo. Considerando os estudos sobre processamento esquemático em Terapia Cognitivo-Comportamental (Knapp \& Beck, 2008) alguns conteúdos 
são mais propensos a ativar determinadas características de personalidade. Neste sentido, estudos que utilizem materiais com maior validade ecológica, como por exemplo, relatos de eventos, poderiam apontar diferentes resultados em termos dos fatores de personalidade.

O presente trabalho aponta para alguns aspectos que parecem avançar em termos dos estudos da interação entre diferenças individuais e falsas memórias. O primeiro aspecto que merece ser destacado refere-se ao fato de que a presença extremamente baixa de um fator de personalidade pode exercer igualmente efeito sobre a suscetibilidade à distorção quanto a presença de altos índices de um determinado fator. $\mathrm{O}$ segundo dado a ser ressaltado associa-se ao fato de que outras características de personalidade, além do neuroticismo já mencionado na literatura, podem exercer papel importante sobre os índices de falsas memórias e de memórias verdadeiras. Por fim, o presente estudo apresenta rara associação explicativa entre dados de pesquisa básica de memória e dados de pesquisa aplicada de estilos cognitivos, tomando por base para suas discussões alguns dos pressupostos teóricos da Terapia Cognitivo-Comportamental. Esta aproximação, que necessita ser aprofundada em estudos posteriores, poderá contribuir significativamente para hipóteses explicativas cada vez mais abrangentes dos erros mnemônicos, indo além da identificação das implicações clínicas, por exemplo, propondo modelos explicativos sobre perfis cognitivos mais suscetíveis a distorções e técnicas de intervenção eficazes para tais indivíduos.

\section{Referências}

Ávila, L. M., \& Stein, L. M. (2006). A influência do traço de personalidade neuroticismo na suscetibilidade às falsas memórias. Psicologia: Teoria e Pesquisa, 22(3), 339-346.

Barbosa, M. E., Ávila, L. M., Feix, L. F., \& Grassi-Oliveira, R. (2010). Falsas memórias e diferenças individuais. In L. M. Stein (Ed.), Falsas memórias: Fundamentos cientificos e suas aplicações clínicas e jurídicas (pp. 133-154). Porto Alegre, RS: Artmed.

Barrett, L. F., Tugade, M. M., \& Engle, R. W. (2004). Individual differences in working memory capacity and dual-process theories of the mind. Psychological Bulletin \& Review, 130(4), 553-573.

Beck, A. T. (1971). Cognition, affect, and psychopathology. Archives of General Psychiatry, 24(6), 495-500.

Beck, A. T., Freeman, A., \& Davis, D. (2005). Terapia cognitiva dos transtornos da personalidade. Porto Alegre, RS: Artmed.

Brainerd, C. J., Stein, L. M., Silveira, R. A. T., Rohenkohl, G., \& Reyna, V. F. (2008). How does negative emotion cause false memories? Psychological Science, 19(9), 919-925.

Drivdahl, S. B., \& Zaragoza, M. S. (2001). The role of perceptual elaboration and individual differences in the creation of false memories for suggested events. Applied Cognitive Psychology, 15, 265-281.

Gallo, D. A. (2010). False memories and fantastic beliefs: 15 years of the DRM illusion. Memory \& Cognition, 38(7), 833-848.

Grassi-Oliveira, R., Gomes, C. F. A., \& Stein, L. M. (2011). False recognition in women with a history of childhood emotional neglect and diagnose of recurrent major depression. Consciousness and Cognition, 20(4), 1127-1134.
Gudjonsson, G. H. (1983). Suggestibility, intelligence, memory recall and personality: An experimental study. The British Journal of Psychiatry, 142, 35-37.

Kensinger, E. A., \& Corkin, S. (2004). Two routes to emotional memory: Distinct neural processes for valence and arousal. Proceedings of the National Academy of Sciences of the USA, 101, 3310-3315.

Knapp, P., \& Beck, A. T. (2008). Fundamentos, modelos conceituais, aplicações e pesquisa da terapia cognitiva. Revista Brasileira de Psiquiatria, 30(Supl. 2), S54-S64.

Neufeld, C. B., Brust, P. B., \& Stein, L. M. (2010). Compreendendo o fenômeno das falsas memórias. In L. M. Stein (Ed.), Falsas memórias: Fundamentos cientificos e suas aplicações clinicas e jurídicas (pp. 21-42). Porto Alegre, RS: Artmed.

Pasquali, L. (2000). Psicometria: Teoria dos testes na Psicologia e na Educação. Petrópolis, RJ: Vozes.

Pasquali, L., Azevedo, M. M., \& Ghesti, I. (1997). Inventário Fatorial de Personalidade: Manual técnico e de avaliação. São Paulo, SP: Casa do Psicólogo.

Peiffer, L. C., \& Trull, T. J. (2000). Predictors of suggestibility and false memory production in young adult women. Journal of Personality Assessment, 74(3), 384-399.

Pervin, L. A., \& John, O. P. (2004). Personalidade: Teoria e pesquisa. Porto Alegre, RS: Artmed.

Reyna, V. F., \& Brainerd, C. J. (1995). Fuzzy Trace Theory: An interim synthesis. Learning and Individual Differences, 7(1), 1-75.

Roediger, H. L., III, \& McDermott, K. B. (1995). Creating false memories: Remembering words not presented in lists. Journal of Experimental Psychology: Learning, Memory, \& Cognition, 21, 803-814.

Salthouse, T. A., \& Siedlecki, K. L. (2007). An individual difference analysis of false recognition. American Journal of Psychology, 120(3), 429-458.

Santos, R. F., Silveira, R. A. T., Gomes, C. F. A., \& Stein, L. M. (2009). Normas de emocionalidade para a versão brasileira do paradigma Deese-Roediger-McDermott (DRM). Psicologia: Teoria e Pesquisa, 25(3), 387-394.

Stein, L. M., \& Gomes, C. F. A. (2009). Normas brasileiras para listas de palavras associadas: Associação semântica, concretude, frequência e emocionalidade. Psicologia: Teoria e Pesquisa, 25(4), 537-546.

Stein, L. M., Feix, L. F., \& Rohenkohl, G. (2006). Avanços metodológicos no estudo das falsas memórias: Construção e normatização do procedimento de palavras associadas. Psicologia: Reflexão e Crítica, 19(2), 166-176.

Stein, L. M., \& Neufeld, C. B. (2001). Falsas memórias: Porque lembramos de coisas que não aconteceram? Arquivos de Ciência Saúde Unipar, 5(2), 179-186.

Stein, L. M., \& Pergher, G. K. (2001). Criando falsas memórias em adultos por meio de palavras associadas. Psicologia: Reflexão e Crítica, 14, 353-366.

Zoellner, L. A., Foa, E. B., Brigidi, B. D., \& Przeworski, A. (2000). Are trauma victims susceptible to "false memories"? Journal of Abnormal Psychology, 109(3), 517-524. 